\title{
Near-infrared electroluminescence from conjugated polymer/lanthanide porphyrin blends
}

\author{
Benjamin S. Harrison, Timothy J. Foley, Mohamed Bouguettaya, James M. Boncella, \\ John R. Reynolds, and Kirk S. Schanze* \\ Department of Chemistry and Center for Macromolecular Science and Engineering, University of Florida, \\ Gainesville, FL 32611-6400 \\ Joonbo Shim and Paul H. Holloway \\ Department of Materials Science and Engineering, University of Florida, Gainesville, FL 32611-6400
}

\section{G. Padmanaban and S. Ramakrishnan}

Department of Inorganic and Physical Chemistry, Indian Institute of Science Bangalore 560012,

Karnataka, India

\begin{abstract}
Near-infrared-emitting polymer light-emitting diodes (PLEDs) have been fabricated using blends of conjugated polymers and lanthanide tetraphenylporphyrin complexes. Host polymers include MEH-PPV and a bis-alkoxy-substituted poly(p-phenylene) (PPP-OR11), and the lanthanide complexes include $\mathrm{Yb}(\mathrm{TPP})$ acac and $\mathrm{Er}(\mathrm{TPP}) \mathrm{acac}$ (where TPP $=5,10,15,20$-tetraphenylporphyrin and $\mathrm{acac}=$ acetylacetonate). Electroluminescence $(\mathrm{EL})$ is observed at $977 \mathrm{~nm}$ from devices fabricated using MEH-PPV or PPP-OR11 blended with $\mathrm{Yb}(\mathrm{TPP}) \mathrm{acac}$, and EL is observed at 1560 $\mathrm{nm}$ from a device fabricated using a blend of MEH-PPV and $\operatorname{Er}(\mathrm{TPP})$ acac. Visible EL from the host polymers is strongly suppressed in all of the devices, however, in the device fabricated using the PPP-OR11 polymer blue emission from the host is completely quenched. Very efficient quenching of the EL from the host in the PPP-OR11 device is believed to occur due to efficient Förster energy transfer, which is facilitated by the excellent spectral overlap between the PPP-OR11 fluorescence and the Soret absorption band of the TPP ligand.
\end{abstract}

Since the initial discovery of polymer organic lightemitting diodes (PLEDs), substantial developments have occurred that have led to a fundamental understanding of their operation as well as the development of practical devices. ${ }^{1}$ Multicomponent polymer and organic LEDs that exploit energy transfer among various emitting chromophores distributed within the active matrix allow precise control of emission energies. These multicomponent devices have been designed using a variety of approaches that include direct blending of two polymers, ${ }^{2}$ the use of intimately mixed polymer layers (such as polyelectrolyte multilayers, thin coated films, laminates, etc.), ${ }^{3-5}$ and the incorporation of emitting metal complexes within polymer host matrices. Examples of the latter include the use of blue-emitting derivatized poly ( $p$ phenylene)s doped with Eu complexes to produce red light emission, ${ }^{6}$ blending of poly( $p$-phenylene vinylene)s with a phosphorescent $\mathrm{Pt}(\mathrm{II})$ - porphyrin, ${ }^{7}$ and luminescent $\operatorname{Ir}(\mathrm{III})$ complexes dispersed in small-molecule host matrices. ${ }^{8}$ Due to their unique electronic structure, many lanthanide ions luminesce in the near IR. Taking advantage of this phenomenon, films of neat lanthanide complexes, a blend of an Er(III) complex in poly(vinylcarbazole), and a blend of a $\mathrm{Nd}$-lissamine complex dispersed in a poly(fluorenebenzothiadiazole) co-polymer host have been used as the emitting materials to afford electroluminescent devices that emit in the near IR. ${ }^{9-11}$ In the present letter, we report

*Electronic mail: kschanze@chem.ufl.edu
near-IR photoluminescence (PL) and electroluminescence (EL) from blends of MEH-PPV or PPP-OR11 with $\mathrm{Yb}$ (TPP)acac and $\operatorname{Er}$ (TPP)acac (Diagram 1, TPP $=5,10,15$, 20 -tetraphenylporphyrin, acac $=$ acetylacetonate). PL and EL in these materials are believed to involve sensitization of the lanthanide-TPP complex by the conjugated polymers, and leads to the narrow-bandwidth emission derived from the $\mathrm{Yb}^{2} \mathrm{~F}_{5 / 2} \rightarrow{ }^{2} \mathrm{~F}_{7 / 2}(977 \mathrm{~nm})$ and $\mathrm{Er}^{4} \mathrm{I}_{13 / 2} \rightarrow{ }^{4} \mathrm{I}_{15 / 2}(1560 \mathrm{~nm})$ transitions.
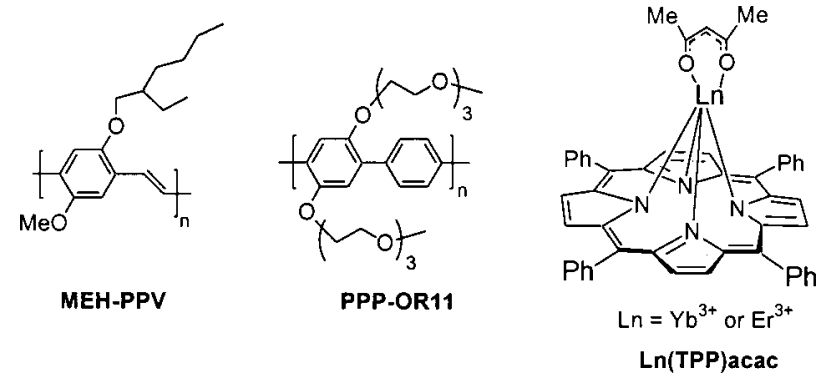

The components of the near-IR emitting devices were selected in order to optimize energy transfer from the conjugated polymer host to the lanthanide-(TPP)acac complexes, and to allow for the most efficient near-IR emission. MEHPPV was selected for the initial work because it is a wellknown and well-characterized material that displays efficient PL and EL. A variety of lanthanides are available to provide tunable PL and EL throughout the near-IR region. Here, we specifically report on $\mathrm{Yb}-$ and $\mathrm{Er}-\mathrm{TPP}(\mathrm{acac})$ complexes, ${ }^{12}$ which provide emission at 977 and $1560 \mathrm{~nm}$, regions that are 


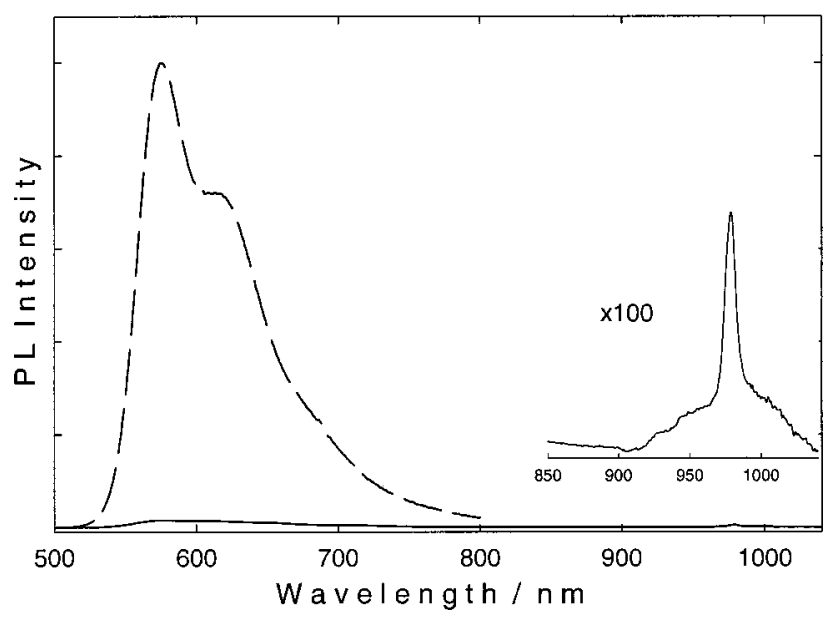

FIG. 1. PL of spin-cast films of neat MEH-PPV (- - -) and $2 \mathrm{~mol} \%$ $\mathrm{Yb}$ (TPP)acac doped in MEH-PPV (-) excited at $350 \mathrm{~nm}$. The spectrum of the blend is plotted on the same absolute scale as that of the neat polymer. The $y$ scale for the inset is expanded by a factor of 100 .

important for optoelectronic communication and biomedical applications.

In order to achieve efficient luminescence from lanthanides, it is necessary to complex the ions with a ligand chromophore that serves to harvest the energy efficiently and sensitize the lanthanide's emission via exchange energy transfer from the ligand-based triplet state. The TPP ligand was deemed to be ideal for this work because of the high degree of spectral overlap of its $Q$-absorption bands with the MEH-PPV fluorescence allowing for highly efficient Förster energy transfer. Due to this excellent spectral overlap, we anticipated that addition of $\mathrm{Yb}(\mathrm{TPP})$ acac or $\operatorname{Er}(\mathrm{TPP})$ acac to MEH-PPV would lead to efficient quenching of the fluorescence from the conjugated polymer host. Furthermore, in lanthanide porphyrin complexes intersystem crossing to the triplet state occurs with $100 \%$ efficiency, thus the ligand is expected to act as an effective sensitizer to produce the spinforbidden, luminescent $\mathrm{F}$ states of the lanthanide ions. PPPOR11 was subsequently selected as a host polymer as it exhibits a high degree of spectral overlap with the Soret band of the TPP ligand.

Formulation of the EL device materials was guided by PL studies of 100-nm-thick spin-coated films produced by blending $\mathrm{Yb}$ (TPP)acac or Er(TPP)acac with MEH-PPV. Figure 1 illustrates the PL of films of neat MEH-PPV and MEH-PPV doped with 2 mol\% Yb(TPP)acac (based on polymer repeat unit). The MEH-PPV fluorescence that appears at $589 \mathrm{~nm}$ is quenched approximately $98 \%$ when $\mathrm{Yb}$ (TPP)acac is present. Quenching of the visible emission is accompanied by the appearance of the $\mathrm{Yb}$ emission at 977 $\mathrm{nm}$ in the near IR. An excitation spectrum for the $977 \mathrm{~nm}$ emission is dominated by the visible absorption of host polymer MEH-PPV, clearly demonstrating its role as a sensitizer. Analogous results are observed when $\operatorname{Er}(\mathrm{TPP})$ acac is blended into MEH-PPV, but in this case the near-IR emission appears at $1560 \mathrm{~nm}$.

The PL observations are consistent with the following sequence of events. Light absorption produces the MEHPPV singlet exciton, which is then trapped by the porphyrin ligand exciting it to the ${ }^{1} \pi, \pi^{*}$ state. Intersystem crossing occurs affording the ${ }^{3} \pi, \pi^{*}$ state of the TPP ligand, which

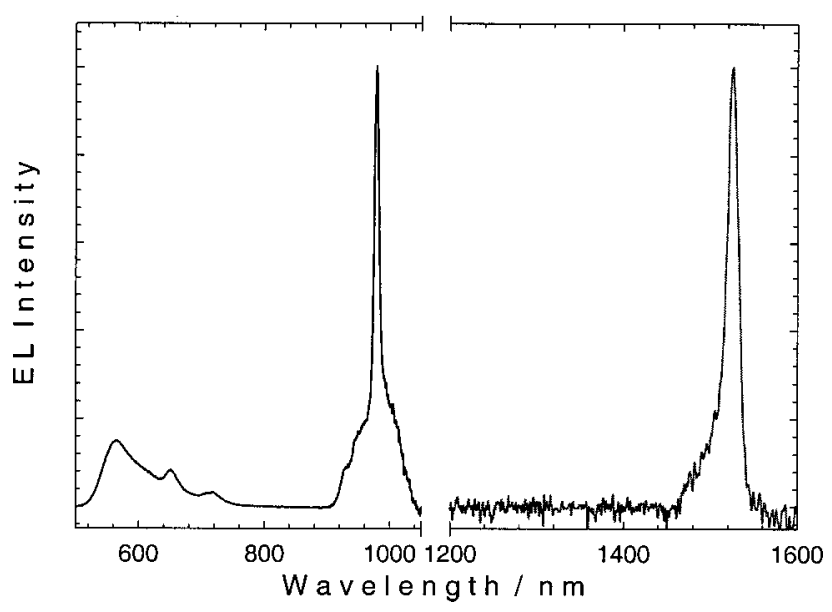

FIG. 2. EL spectrum of MEH-PPV doped with 5 mol \% Yb(TPP)acac measured at $9 \mathrm{~V}$ (left). EL spectrum of $5 \mathrm{~mol} \% \mathrm{Er}(\mathrm{TPP}) \mathrm{acac}$ at $13 \mathrm{~V}$ is shown on the right. The visible emission is not shown but is similar in intensity to the $\mathrm{Yb}$ (TPP)acac doped device. The two bands on the red side of the MEH-PPV emission are believed to arise from a free base TPP impurity.

subsequently sensitizes the $\mathrm{Yb}^{2} \mathrm{~F}_{5 / 2}$ state which emits at 977 nm.

Near-IR electroluminescent devices were constructed beginning with indium-tin-oxide (ITO) glass coated with PEDOT/PSS (Bayer Baytron P VP Al 4083) as a hole transport layer. The MEH-PPV:Ln(TPP)acac blend was spin coated from solution ( $1 \mathrm{wt} \%$ of the polymer in toluene) and the resulting film was vacuum dried for $12 \mathrm{~h}$ (1 $\times 10^{-6}$ Torr) at room temperature. Calcium (50 $\AA$ ) followed by $\mathrm{Al}\left(\begin{array}{ll}1500 & \AA\end{array}\right)$ layers were thermally evaporated at 1 $\times 10^{-6}$ Torr without breaking the vacuum between metal depositions. After deposition, the device was encapsulated with epoxy to minimize exposure to oxygen and moisture. All device measurements were made at room temperature.

Figure 2 shows the EL spectra of PLEDs fabricated using the $\mathrm{Ln}(\mathrm{TPP})$ acac complexes where $\mathrm{Ln}=\mathrm{Yb}^{3+}(5 \mathrm{~mol} \%)$ and $\mathrm{Er}^{3+}(5 \mathrm{~mol} \%)$. The spectral data show that the visible emission of MEH-PPV at $589 \mathrm{~nm}$ is strongly suppressed, and the device emission is dominated by the near-IR output of the Ln complexes. The data show that the narrowbandwidth characteristic of the lanthanide emission is preserved in the EL devices.

Figure 3 shows the room-temperature $I-V$ and quantitative light output characteristics for a device that contains 5 mol \% of $\mathrm{Yb}(\mathrm{TPP})$ acac blended with MEH-PPV as the active material. It can be seen that the emission turns on at $\sim 4$ $\mathrm{V}$ and the device exhibits typical Schottky diode characteristics. The turn-on voltage is low compared to devices constructed with neat lanthanide complexes. ${ }^{13,14}$ At high current densities, a decrease in the emission efficiency is observed. The basis for the decrease in device efficiency at high voltage is unknown at the present time and is the focus of an ongoing investigation. The external near-IR EL efficiency of the device is $1.5 \times 10^{-4}$ at $7 \mathrm{~V}$. The comparatively low device efficiency is due to highly efficient nonradiatative decay channels that operate in the lanthanide excited state. The fact that nonradiative decay is an important decay channel to the $\mathrm{Yb}{ }^{2} F_{5 / 2}$ state in the blends is confirmed by temperaturedependent PL experiments which show that the intensity of the $977 \mathrm{~nm}$ emission increases with decreasing temperature. 


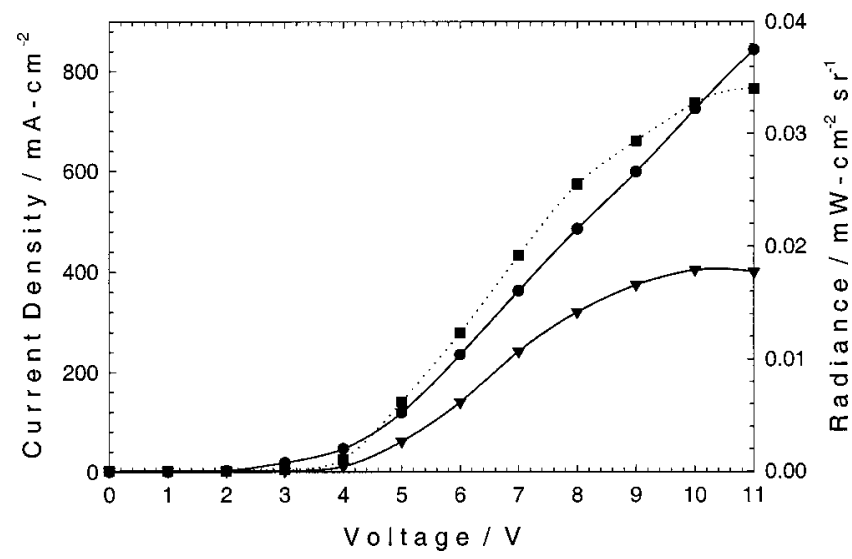

FIG. 3. Current ( ) and power output of visible $(\boldsymbol{\nabla})$ and near-infrared regions of $5 \mathrm{~mol} \% \mathrm{Yb}(\mathrm{TPP})$ acac in $\mathrm{MEH}-\mathrm{PPV}$ as a function of increasing voltage at room temperature.

Early results indicate that encapsulated device lifetimes of greater than $24 \mathrm{~h}$ are obtained with the $\mathrm{Yb}^{3+}$ systems and can be easily enhanced.

In order to demonstrate the generality of the approach of using blends of Ln(TPP)acac complexes with conjugated polymers as the active material in near-IR PLEDs, a device was fabricated that utilizes the blue-emitting alkoxy substituted poly( $p$-phenylene) PPP-OR $11^{15}$ (Diagram 1) as the host polymer blended with $\mathrm{Yb}(\mathrm{TPP})$ acac $(20 \mathrm{~mol} \%)$. The PPP-OR11:Yb(TPP)acac device was fabricated according to the same procedures described above, except that a more concentrated polymer solution was used for spin coating (2 wt $\%$ in chloroform). Figure 4 shows that the EL of the PPPOR11:Yb(TPP)acac device is dominated by the $977 \mathrm{~nm} \mathrm{Yb}$ emission. Near-IR EL from the PPP-OR11:Yb(TPP)acac device turns on at $4 \mathrm{~V}$ and it features an external quantum efficiency of $1.0 \times 10^{-3}$ at $7 \mathrm{~V}$, which is more than five-fold higher compared to the MEH-PPV:Yb(TPP)acac device. Importantly, EL is not observed from the PPP-OR11 host in the 400-600 nm region, although a very weak red emission is observed which is believed to arise from free base TPP which is present as a trace impurity. The lack of EL from the PPP-OR11 host indicates that singlet excitons that are produced on the polymer are effectively quenched by $\mathrm{Yb}(\mathrm{TP}-$ P)acac. Efficient quenching is believed to occur because of the excellent spectral overlap between the PPP-OR11 emission $\left(\lambda_{\max } \approx 410 \mathrm{~nm}\right)$ and the TPP Soret band $\left(\lambda_{\max }\right.$ $\approx 420 \mathrm{~nm})$.

We are expanding on these initial observations by fabricating PLEDs that use a range of conjugated polymer hosts and lanthanide complexes. These devices will produce EL that can be tuned across the near-IR region. A complete account of this work will appear in the near future.

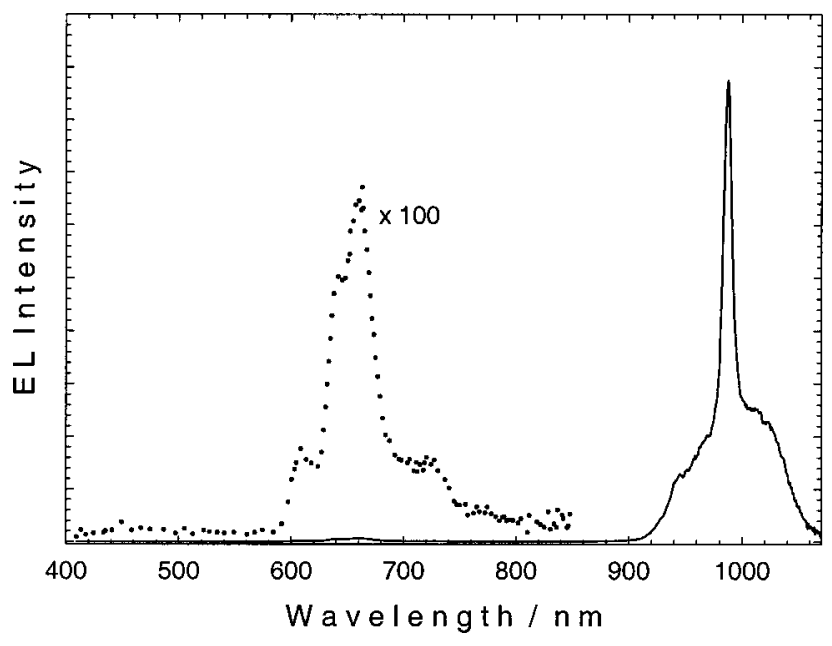

FIG. 4. EL spectrum of PPP-OR11 doped with 20 mol \% Yb(TPP)acac measured at $9 \mathrm{~V}$. The dotted line shows the visible region with the $y$ scale expanded by a factor of 100 . The weak EL that appears in the 600-700 nm region is believed to arise from the TPP triplet state.

This work was supported by the Defense Advanced Research Projects Agency (Grant No. DAAD 19-00-1-0002).

${ }^{1}$ J. H. Burroughes, D. D. C. Bradley, A. R. Brown, R. W. Marks, K. Mackay, R. H. Friend, P. L. Burn, and A. B. Holmes, Nature (London) 347, 539 (1990); D. Braun and A. J. Heeger, Appl. Phys. Lett. 58, 1982 (1991); A. Kraft, A. C. Grimsdale, and A. B. Holmes, Angew. Chem. Int. Ed. Engl. 37, 402 (1998).

${ }^{2}$ M. Granström and O. Inganäs, Adv. Mater. 7, 1012 (1995).

${ }^{3}$ M. Granström, K. Petritsch, A. C. Arias, A. Lux, M. R. Andersson, and R. H. Friend, Nature (London) 395, 257 (1998).

${ }^{4}$ J. W. Baur, S. Kim, P. B. Balanda, J. R. Reynolds, and M. F. Rubner, Adv. Mater. 10, 1452 (1998)

${ }^{5}$ S.-C. Chang, J. Bharathan, Y. Yang, R. Helgeson, F. Wudl, M. Ramey, and J. R. Reynolds, Appl. Phys. Lett. 73, 2561 (1998).

${ }^{6}$ M. D. McGehee, T. Bergstedt, C. Zhang, A. P. Saab, M. B. O’Regan, G. C. Bazan, V. I. Srdanov, and A. J. Heeger, Adv. Mater. 11, 1349 (1999).

${ }^{7}$ V. Cleave, G. Yahioglu, P. Le Barny, D. Hwang, A. B. Holmes, R. H. Friend, and N. Tessler, Adv. Mater. 13, 44 (2001).

${ }^{8}$ R. C. Kwong, S. Lamansky, and M. E. Thompson, Adv. Mater. 12, 1134 (2000); S. Lamansky, P. Djurovich, D. Murphy, F. Abdel-Razzaq, H.-E. Lee, C. Adachi, P. E. Burrows, S. R. Forrest, and M. E. Thompson, J. Am. Chem. Soc. 123, 4304 (2001).

${ }^{9}$ R. G. Sun, Y. Z. Wang, Q. B. Zheng, H. J. Zhang, and A. J. Epstein, J. Appl. Phys. 87, 7589 (2000).

${ }^{10}$ Y. Kawamura, Y. Wada, and S. Yanagida, Jpn. J. Appl. Phys., Part 1 40, 350 (2001).

${ }^{11}$ L. H. Slooff, A. Polman, F. Cacialli, R. H. Friend, G. A. Hebbink, F. C. J. M. van Veggel, and D. N. Reinhoudt, Appl. Phys. Lett. 78, 2122 (2001).

${ }^{12}$ W. D. Horrocks and C. P. Wong, J. Am. Chem. Soc. 98, 7157 (1976).

${ }^{13}$ Y. Kawamura, Y. Wada, M. Iwamuro, T. Kitamura, and S. Yanagida, Chem. Lett. 3, 280 (2000).

${ }^{14}$ R. J. Curry and W. Gillin, Synth. Met. 111, 35 (2000); Appl. Phys. Lett. 75, 1380 (1999)

${ }^{15}$ P. B. Balanda, Ph.D. dissertation, University of Florida (1997). 